\title{
ESTUDIO DE CAPACIDAD DE CARGA AMBIENTAL JARDÍN BOTÁNICO UNIVERSIDAD TECNOLÓGICA DE PEREIRA
}

\author{
JORGE HUGO GARCÍA SIERRA ${ }^{1}$ Y JESICA LEANDRA RAMÍREZ CARDONA ${ }^{2}$ \\ jorgehugo@utp.edu.co ; jlramirez@utp.edu.co
}

Manizales, 2010-08-12 (Rev. 2010-12-14)

\section{RESUMEN}

Los estudios de capacidad de carga ambiental han sido desarrollados, tanto a nivel internacional como nacional, principalmente en áreas protegidas, pero su aplicación en los jardines botánicos asume igual importancia al propiciar de la misma forma que en las primeras, el establecimiento de criterios que promuevan una armonía entre el cumplimiento de los objetivos de conservación con los relativos al ecoturismo y la educación ambiental.

Desde esta perspectiva, con el desarrollo de este estudio se pretende contribuir al cumplimiento armónico de los objetivos del Jardín Botánico Universidad Tecnológica de Pereira (JB-UTP), generando además una actualización a la "Determinación de la Capacidad de Carga Turística", proceso que se había realizado en el año 2002.

Se intenta exponer en este sentido, una breve contextualización teórico-normativa del tema, así como una específica del área de estudio con el fin de aproximarse a un mayor conocimiento y entendimiento de la situación que se pretende abordar. Ya en la parte metodológica se retoman los aportes de la "Metodología de Capacidad de Carga Turística", complementada con otra conceptualizaciones sobre aspectos ecosistémicos y administrativos; así como con un mayor rigor técnico y objetividad.

Se presentan entonces tres fases: en primera instancia una turística, que tiene en cuenta la capacidad física en términos de tiempo y espacio para las actividades ecoturísticas, y a la vez unos factores de corrección relacionados con la satisfacción del visitante; en segundo lugar, una que tiene en cuenta la resiliencia ecosistémica aplicando de igual forma factores de corrección alusivos, y finalmente, una ambiental que fusiona las dos anteriores retomando la capacidad institucional del JB-UTP para la gestión y manejo del área.

\section{PALABRAS CLAVE:}

Capacidad de carga ambiental, Jardín Botánico, resiliencia ecosistémica, capacidad de gestión, turismo.

\section{STUDY OF ENVIRONMENTAL LOAD CAPACITY UNIVERSIDAD TECNOLOGICA DE PEREIRA BOTANICAL GARDEN}

\section{ABSTRACT}

The environmental load capacity studies have been developed both nationally and internationally mainly in protected areas but their application in botanical gardens assumes equal importance while bringing about, in the same way as in the first ones, the establishment of criteria that promotes harmony between the fulfillment of conservation objectives with those related to eco-tourism and environmental education.

From this perspective, with the development of this study, it is intended to contribute to the harmonic fulfillment of the objectives at Universidad Pedagógica de Pereira Botanical Garden (UTP- BG) thus generating an update to the "Determination of the tourist load capacity", process that had been carried out in 2002.

The study attempts to present in this way, a brief theoretical and normative contextualization of the topic, as well as a specific contextualization of the area of study with the purpose of approaching to a greater knowledge and understanding of the situation that wants to be dealt with. From the methodological viewpoint, the contributions from the "Tourism Load Capacity Methodology" are taken up complemented with another conceptualization about eco-systemic and administrative aspects and using a greater objectiveness rigor. 
Three phases are presented: firstly the touristic phase that takes into consideration the physical capacity in terms of time and space for eco-touristic activities and some correction factors related with the visitors' satisfaction; secondly, one taking into consideration the eco-systemic resilience also applying the respective correction factors, and finally an environmental one which puts together the previous two , retaking the UTP-BG institutional capacity for management and operation of the area.

\section{KEY WORDS:}

Environmental load capacity, Botanical Garden, ecosystems resilience, management capacity, tourism.

\section{ANTECEDENTES}

Según Rome (1999):

El concepto y la práctica del monitoreo de los visitantes de las zonas protegidas han regido en los Estados Unidos durante 25 años, por lo menos, pero en América Latina el advenimiento del turismo basado en la naturaleza es relativamente reciente y hasta hace poco, ha sido escasa la necesidad de métodos de limitación de los impactos del turismo o los incentivos para crearlos. (p. 5)

Sin embargo, Rome (1999) habla de que pese a que el ecoturismo hoy día tenga un gran auge en los países latinoamericanos, son necesarias metodologías que se ajusten a las realidades propias, que por cierto, difieren en alto grado de las estadounidenses; principalmente en términos de recursos presupuestarios y técnicos sustanciales, en la necesaria armonía entre conservación y desarrollo que debe primar para América Latina, así como en la particularidad que tienen las áreas protegidas de esta región respecto a las poblaciones que generalmente habitan o se vinculan de alguna forma con estas zonas de conservación natural.

A nivel mundial se reconocen diferentes metodologías que evalúan la capacidad de carga y/o establecen indicadores y mecanismos de seguimiento y monitoreo en áreas protegidas, tal como dicen Cifuentes et al. (1999) "se destacan el VIM 'Visitor Impact Management' (Loomis y Graefe, 1992), LAC 'Límites de cambio aceptable' (Stankey et al., 1985), y CCT 'Capacidad de Carga Turística' (Cifuentes, 1992)" (p. 6).

En el orden nacional si bien se optó por concesionar los servicios ecoturísticos que pueden prestar las áreas protegidas, según la Contraloría General de la República (2007) "esto no se dispuso de acuerdo con una caracteriาzación de línea base debidamente estructurada, que sirviera de referente para soportar evaluaciones periódicas, ni de ejercicios rigurosos y más detallados de evaluación de la capacidad de carga de dichas áreas" (p. 372); lo que demuestra que son aún incipientes los procesos relacionados con el manejo de visitantes en armonía con la conservación de la biodiversidad.

Ya a nivel departamental (Risaralda), se tiene la "Determinación de la capacidad de carga ecoturística como herramienta de planificación y manejo del Santuario de Fauna y Flora Otún Quimbaya - Vereda La Suiza, Municipio de Pereira" realizado en el año 2003, desarrollo que se aportó desde el programa de Administración del Medio Ambiente de la Facultad de Ciencias Ambientales de la Universidad Tecnológica de Pereira -UTP- mediante la elaboración de un proyecto de grado.

Sin embargo, las experiencias antes mencionadas se han centrado principalmente en áreas protegidas, y no se tienen por lo menos, evidencias escritas y difundidas de logros en el tema por parte de los jardines botánicos. En la justificación del presente estudio se expone claramente la necesidad de que los jardines botánicos, como áreas de conservación de la flora nativa de una región y como facilitadores de procesos educativos ambientales y ecoturísticos, requieren al igual que las áreas protegidas, de criterios debidamente soportados para el manejo óptimo y en armonía con la preservación.

Los pocos avances que se tienen al respecto son alusivos al Jardín Botánico de Bogotá "José Celestino Mutis", el cual posee un estudio de capacidad de carga ${ }^{3}$; y así mismo, el Jardín Botánico Universidad Tecnológica de Pereira, fue sujeto de un estudio denominado "Determinación de la capacidad de carga y diseño de la propuesta de interpretación ambiental para el Jardín Botánico de la Universidad Tecnológica de Pereira" realizado en el año 2003 como resultado de un proyecto de grado elaborado también desde el programa de Administración del Medio Ambiente de la UTP. Se 
caracterizan entonces estas dos experiencias como las únicas conocidas hasta el momento y dentro de las posibilidades del presente estudio para el país.

\section{ASPECTOS TEÓRICO-NORMATIVOS}

\section{Jardines Botánicos}

Según Wyse y Sutherland (2000):

Existen más de 1800 jardines botánicos y arboreta en 148 países del mundo y éstos mantienen más de 4 millones de accesiones de plantas vivas (cada una de las plantas coleccionadas). En estas colecciones hay representantes de más de 80.000 especies, casi un tercio de las especies de plantas vasculares del mundo. Estas colecciones tienen una amplia diversidad de plantas y son particularmente ricas en algunos grupos como orquídeas, cactus y otros grupos suculentos, palmas, bulbos, coníferas, árboles y arbustos de zonas templadas y especies silvestres, en especial aquellas que se encuentran amenazadas.

También poseen decenas de miles de especies cultivadas de importancia económica y sus parientes silvestres, como los árboles frutales y plantas medicinales. Adicionalmente, los jardines botánicos cuentan con muchas otras colecciones como son los herbarios (especies preservadas) y bancos de semillas. (p. 16)

\section{Capacidad de Carga Ambiental}

Se retoma la definición de capacidad de carga turística ya que éste es un tipo específico de capacidad de carga ambiental, el cual se refiere a la capacidad biofísica y social del entorno respecto de la actividad turística y su desarrollo. El término representa también el máximo nivel de uso por visitantes que un área puede albergar, y se puede definir como la capacidad que posee un ecosistema para mantener organismos mientras mantiene su productividad, adap 7 tabilidad y capacidad de regeneración, es decir, esboza el límite de la actividad humana: pues si éste es excedido, el recurso se deteriorará (Ceballos-Lascuráin, 1996 citado en Cifuentes et al., 1999, p. 6).

Pero en síntesis, se desea fundamentar el concepto con base en la significación del ambiente, pues si bien éste se define como un tipo de capacidad de carga ambiental, en el presente estudio pretende configurarse a partir de la comprensión de la interacción ecosistema (zona de conservación) y cultura (educación ambiental y ecoturismo) ${ }^{4}$ y lo que ésta supone. En un sentido más amplio se plantearía capacidad de carga ambiental en lugar de capacidad de carga turística, puesto que así se asumiría concretamente el contexto de relaciones ambientales que se derivan de ejecutar procesos de educación ambiental en áreas destinadas a la conservación, y la emergencia en el sentido de otorgar un manejo ambiental soportado en el conocimiento de estas relaciones.

\section{Capacidad de Gestión}

Se tiene en cuenta el concepto de capacidad de manejo propuesta por Cifuentes, Izurieta y De Faria (2000), quienes definen el manejo:

como el conjunto de acciones de carácter político, legal, administrativo, de investigación, planificación, protección, coordinación, promoción, interpretación y educación, entre otras, que dan como resultado el mejor aprovechamiento y la permanencia de un área protegida, y el cumplimiento de sus objetivos. (p. 5)

No obstante, se considera pertinente abarcar lo anterior desde los conceptos básicos de la administración-gestión, pues puede ser una noción más integradora frente a los componentes del manejo antes mencionados, agrupándolos en tres aspectos clave: planificación, ejecución y control. En este orden de ideas, la gestión facilitaría la comprensión y manejo sobre los aspectos positivos y negativos derivados de la relación ambiental área de conservación natural (ecosistema) actividades turístico-educativas (cultura). 


\section{Ley 299 de 1996}

Habla lo pertinente a que los jardines botánicos deberían ejecutar programas permanentes de educación, implicando con ello procesos de manejo de visitantes en estas áreas de conservación de la flora.

\section{Plan Nacional para Jardines Botánicos de Colombia (1998)}

Plantea la visión y misión de los jardines botánicos en el país. Así mismo, expone diez estrategias principales relacionadas con:

- El fortalecimiento de la capacidad administrativa; de la labor de la conservación de la flora amenazada; de las actividades de educación ambiental para contribuir hacia la generación de una conciencia sobre la importancia de la flora, y de las relaciones y vínculos con otras entidades a través de la Red Nacional de Jardines Botánicos de Colombia.

- La promoción de actividades de investigación sobre la flora.

- La capacitación de los funcionarios de los jardines botánicos.

- El aumento de la representatividad de los jardines.

- El desarrollo de los sistemas de información y su integración con el Sistema Nacional Ambiental -SINA-

- La divulgación de la normatividad vigente sobre la flora y los jardines.

- Y finalmente, el diseño e implementación de la estrategia de sostenibilidad financiera.

\section{Ley 300 de 1996}

Define el concepto de ecoturismo como "una forma de turismo especializado y dirigido que se desarrolla en áreas con un atractivo natural especial y se enmarca dentro de los parámetros del desarrollo humano sostenible", y de igual forma define la capacidad de carga como el:

nivel de aprovechamiento turístico (número de personas) que una zona puede soportar asegurando una máxima satisfacción a los visitantes y una mínima repercusión sobre los recursos naturales y culturales. Esta noción supone la existencia de límites al uso, determinada por factores medioambientales, sociales y de gestión que define la autoridad ambiental.

\section{Política para el Desarrollo del Ecoturismo (2003)}

Habla respecto al concepto de la capacidad de carga en relación con dos de las líneas estratégicas de la política:

En primer lugar, el "ordenamiento y planificación de las áreas", en donde se plantea que la capacidad de carga ecológica y material debe ser un criterio para su desarrollo, pues se deben establecer medidas de control de uso de las áreas de tal manera que no se llegue a generar deterioro de los recursos. Estas medidas se pueden sustentar en la aplicación de metodologías ampliamente reconocidas, como las tradicionales de medición de Capacidad de Carga Turística (CCT), la metodología VIM (Visitors Impact Management) que, junto a los Límites de Cambio Aceptable (LCA), son opciones que deben escoger las regiones de acuerdo con las características del sitio, con la disponibilidad de los recursos para aplicar las medidas respectivas y con la capacidad técnica (conocimientos) de que se disponga en el área.

Y en segundo orden, la "investigación de mercados y el diseño del producto", en donde se esboza que para el diseño del producto se deben programar actividades soportadas en estudios de capacidad de carga; además se debe tener en cuenta que si es necesario desarrollar los servicios de apoyo de las actividades ecoturísticas, esto debe hacerse sin sobrepasar la capacidad de carga del entorno natural y cultural.

\section{ÁREA DE ESTUDIO}

Según el Jardín Botánico Universidad Tecnológica de Pereira (2007) aunque el área oficial de este Jardín Botánico es 12,7 ha, la Universidad Tecnológica de Pereira posee 17,4 ha adicionales en bosque natural, siendo en total el $61 \%$ del área total del campus. Así mismo, este Jardín es 
categorizado como "Natural o Silvestre" según la BGCI, lo que lo hace ser muy diferente a los que se encuentran en las principales ciudades de Colombia, Estados Unidos o Europa, ya que la conservación no se realiza por medio de colecciones botánicas ornamentales, hortícolas o forestales diseñadas, sino en un área de vegetación que ha surgido naturalmente y está bajo su manejo, protección y enriquecimiento. (pp. 7-8).

Figura 1. Ubicación UTP en el casco urbano del municipio de Pereira.

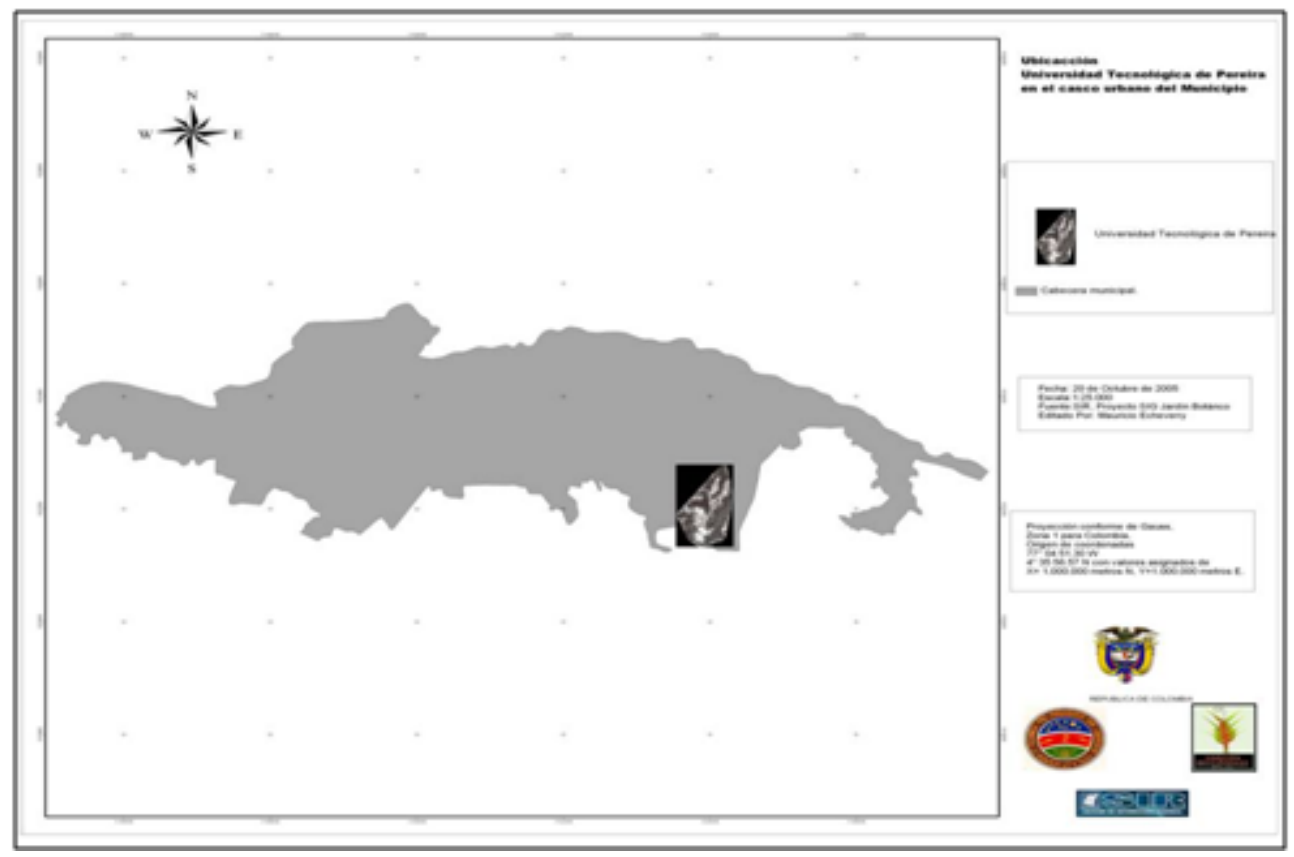

Fuente: Echeverry (2005).

De acuerdo con el Grupo de Estudio en Fauna Silvestre Universidad de Caldas (2005), en la zona se pueden apreciar tres diferentes ecosistemas: Bosque de Guadua, Bosque secundario en diferentes estados sucesionales (Bosque Andino) y un humedal. Además, del área total del Jardín Botánico (12,7 ha) 10,9 corresponde a áreas boscosas donde predominan especies como Cedro negro (Juglans neotropica), Chaquito (Podocarpus oleifolium), Cedro de Montaña (Cedrela montana), Comino (Aniba perutilis), Sande (Brosimum utile), Cattleya quadricolor y Cattleya shoeredae y la altura máxima de los árboles no excede los 20 metros, Guaduales con edades aproximadas a 20 años y alturas promediadas de 30 metros; 1,7 hectáreas corresponden al humedal formado artificialmente pero alimentado por numerosos nacimientos en su mayoría provenientes de la zona de los Guaduales. (p. 5).

Jardín Botánico y Campus Universidad Tecnológica de Pereira (2005) hablan de que:

En el inventario preliminar de especies vegetales del Jardín Botánico se cuantifican 263 especies dentro del área boscosa, a las que se suman las nuevas especies de las colecciones presentes en el vivero, producto del enriquecimiento constante que se ha desarrollado desde mediados del año 2004. Así mismo, a la fecha se han identificado 168 especies de aves (Momotus momota, Bubulcus ibis, Pionus menstruus, Florisuga mellivora, Phaetornis guy, Aulacorhynchus haematopygus, Campephilus pollens, Dryccopus lineatus) de las cuales 15 son migratorias, 15 de reptiles (Iguana iguana, Anolis tropidogaster, Leptodeira septentrionalis, Trachemys scripta, Kinosternon scorpioides), 7 de mamíferos (Didelphys marsupialis, Didelphys albiventris, Sciurus granatensis, Vampirum spectrum, Potos flavus) y 3 de anfibios (Bufo marinus, Hyla microcephala). (p. 4)

Ya en lo relacionado con los aspectos administrativos y misionales, Quintana (2005) plantea que:

Los procesos desarrollados por el JB-UTP que generan resultados para ser ofrecidos a clientes o usuarios externos a la organización en términos de productos y servicios se denominan procesos operativos o misionales, los procesos cuyos resultados son utilizados por la organización para dar soporte administrativo a las acciones desarrolladas por la misma se denominan procesos de apoyo, 
y los procesos cuyos resultados trazan el rumbo y las estrategias para el funcionamiento general del JB-UTP se denominan: procesos estratégicos. (p. 46)

Figura 2. Mapa de procesos JB-UTP

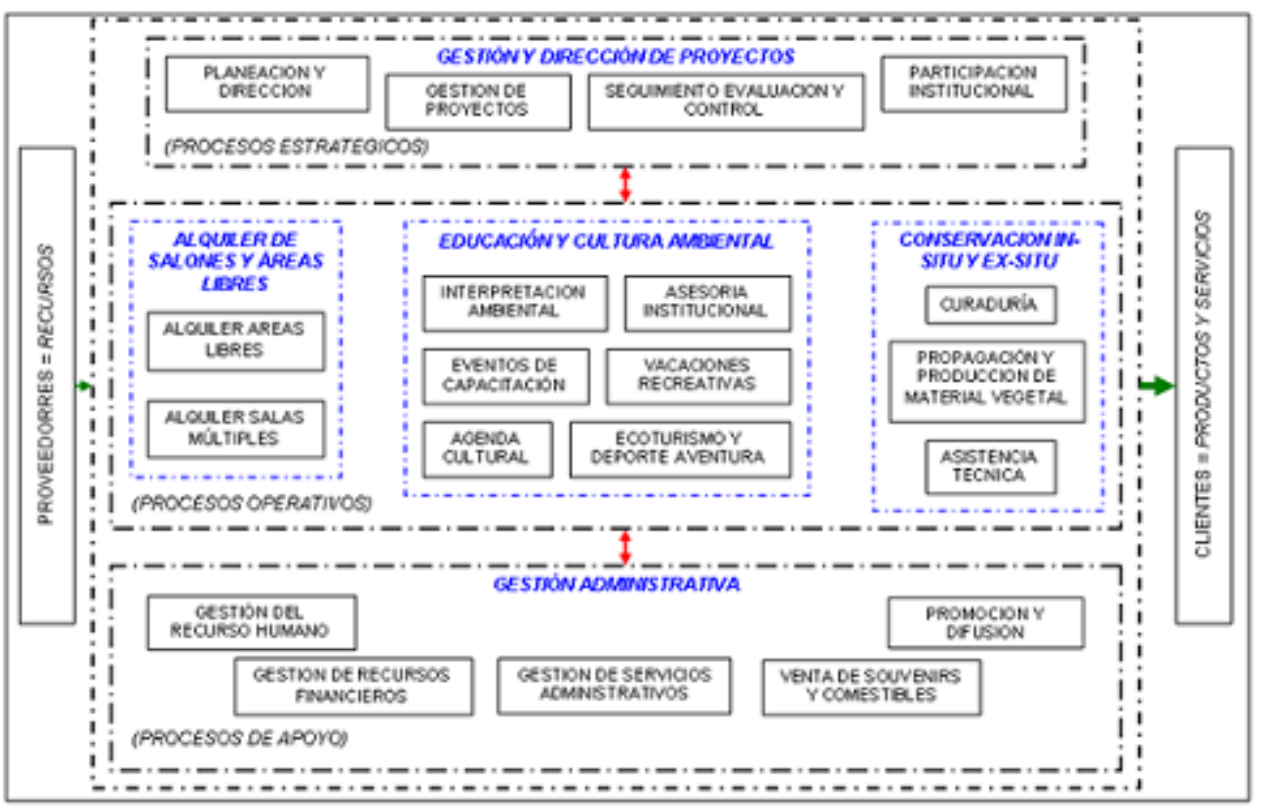

Fuente: Quintana, R. (2009) - Bases para la implementación de un sistema de gestión ambiental para el Jardín Botánico Universidad Tecnológica de Pereira.

En la Figura 3 se exponen los circuitos de interpretación ambiental que se manejan en el Jardín Botánico Universidad Tecnológica de Pereira en el marco del Programa de Educación Ambiental, los cuales serán objeto directo de análisis y procesos de cálculo en el presente estudio, para determinar la capacidad máxima de visitantes permitidos en un tiempo dado, sin irse en detrimento del ecosistema, garantizando la satisfacción del visitante y fundamentándose en la actual capacidad administrativa del JB-UTP.

Figura 3. Circuitos de Interpretación Ambiental JB-UTP.

\begin{tabular}{|c|c|c|c|}
\hline Circuito & Senderos & Duración del recorrido & Especificaciones \\
\hline 1 & $\begin{array}{l}\text { Rizomas - Barranquero } \\
\text { Guadua B - Guadua }\end{array}$ & 30 minutos & $\begin{array}{l}\text { Se realiza para niffos de pre. } \\
\text { jardin, personas de la tercera } \\
\text { edad o euando se requiere un } \\
\text { recorrido corto }\end{array}$ \\
\hline 2 & $\begin{array}{l}\text { Rizomas - Barranquero - Arrieral } \\
\text { - Humedal A - Caminante B - } \\
\text { Guadua A (hasta la puerta del } \\
\text { Planetario) }\end{array}$ & 1 hora & $\begin{array}{l}\text { Para recorrdo corto, cuando los } \\
\text { vistantes han programado ida a } \\
\text { Planetario de la UTP y deben } \\
\text { salir la puerta que comunica el } \\
\text { JB directamente con este. }\end{array}$ \\
\hline 3 & $\begin{array}{l}\text { Rizomas - Barranquero - Arrieral } \\
\text { - Humedal A - Caminante B . } \\
\text { Guadua A }\end{array}$ & 1 hora y media & $\begin{array}{l}\text { Recorrido mas completo por los } \\
\text { senderos del JB }\end{array}$ \\
\hline 4 & $\begin{array}{l}\text { Rizomas - Barranquero - Arrieral } \\
\text { - Humedal B - Caminante B - } \\
\text { Guadua A }\end{array}$ & 2 horas & $\begin{array}{l}\text { Recorrido más completo por los } \\
\text { senderos del JB }\end{array}$ \\
\hline
\end{tabular}

Fuente: elaboración propia. 


\section{PROCESO METODOLÓGICO}

Figura 4. Esquama metodologloo caloulo de Capacidad de Carga Amolental (CCA).

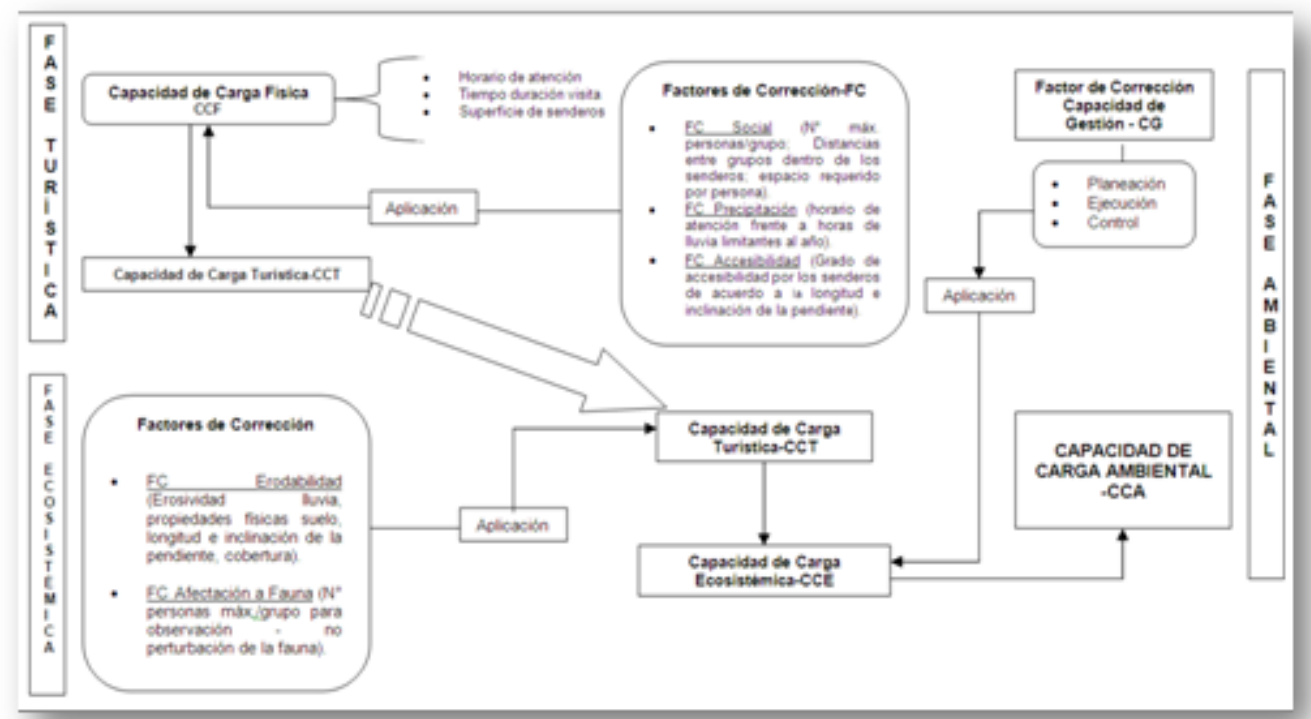

Fuente' elaboracion propla.

\section{Cálculo de Capacidad de Carga Ambiental}

El proceso de cálculo de la Capacidad de Carga Ambiental (CCA) se divide en tres niveles consecutivos, que se caracterizan por ser cada uno un factor de corrección del anterior:

Cálculo de Capacidad de Carga Turística (CCT).

Cálculo de Capacidad de Carga Ecosistémica (CCE).

Cálculo de Capacidad de Gestión (CG).

Así, los tres niveles de capacidad tienen una relación que puede representarse como sigue:

\section{$\mathrm{CCT} \geq \mathrm{CCE} \geq \mathrm{CCA}$}

\section{Cálculo de Capacidad de Carga Turística (CCT)}

Es el límite máximo de visitas que se pueden hacer al sitio durante un día. Está dada por la relación entre factores de visita (horario y tiempo de visita), el espacio disponible y la necesidad de espacio por visitante. Para el cálculo se utilizó la siguiente fórmula:

$$
\mathrm{CCF}=(S / S p) \times N V
$$

Donde:

CCF = Capacidad de Carga Física.

$\mathrm{S}=$ Superficie disponible, en metros lineales.

$\mathrm{sp}=$ Superficie usada por persona $=1 \mathrm{~m}$ de sendero .

NV = Número de veces que el sitio puede ser visitado por la misma persona en un día.

Para estimar NV se debe utilizar la siguiente fórmula: 


\section{$\mathrm{NV}=\mathrm{Hv} / \mathrm{tv}$}

Donde:

Hv = Horario de visita.

tv = tiempo necesario para visitar cada sendero.

No obstante, los anteriores cálculos requieren de la aplicación de factores de corrección, que se deben estimar a partir de la siguiente fórmula general:

$$
\mathrm{FCx}=1-(M l x / M t x)
$$

Donde:

FCx $=$ Factor de Corrección por la variable " $x$ ".

$\mathrm{Mlx}=$ Magnitud limitante de la variable " $\mathrm{x}$ ".

$M t x=$ Magnitud total de la variable " $x$ ".

\section{Factor de Corrección Social (FCsoc)}

Considerando aspectos referentes a la calidad de visitación, se plantea la necesidad de manejar la visitación por grupos. Para un mejor control del flujo de visitantes, y a la vez asegurar la satisfacción de éstos, se propone que la visitación sea manejada bajo los siguientes supuestos ${ }^{5}$ :

- Grupos de máximo 26 personas.

- La distancia entre grupos debe ser de al menos $30 \mathrm{~m}$, para evitar interferencias entre grupos. Así, dada la distancia mínima entre grupos requerida sumado a que cada persona ocupa $1 \mathrm{~m}$ de sendero, cada grupo requeriría de $56 \mathrm{~m}$.

El número de grupos que puede estar simultáneamente en cada sendero se calcula así:

$$
N G=S / D G
$$

Donde:

NG = Número de Grupos.

$S=$ Superficie disponible en metros lineales.

DG = Distancia requerida por cada grupo para movilizarse dentro del sendero adecuadamente.

Para calcular el factor de corrección social es necesario primero identificar cuántas personas pueden estar simultáneamente dentro de cada sendero.

Esto se hace a través de

$$
P=N G \times N p G
$$

Donde:

$\mathrm{P}=$ Personas que pueden estar simultáneamente en cada sendero.

$\mathrm{NpG}=$ Número de personas permitidas por Grupo.

Para calcular el Factor de Corrección Social (FCsoc) necesitamos identificar la magnitud limitante que, en este caso, es aquella porción del sendero que no puede ser ocupada porque hay que mantener una distancia mínima entre grupos. Por esto, dado que cada persona ocupa $1 \mathrm{~m}$ del sendero, la magnitud limitante es igual a:

$$
m l \text { FCsoc }=m t-P
$$


Donde:

$\mathrm{ml} \mathrm{FCsoc}=$ magnitud limitante .

$\mathrm{mt}=$ magnitud total (en este caso es la superficie total disponible del circuito).

$\mathrm{P}=$ Personas que pueden estar simultáneamente en cada circuito.

Entonces:

$$
F C \operatorname{soc}=1-(m l / m t)
$$

Factor de Corrección Precipitación (FCpre)

La siguiente es la fórmula en la cual se basa el cálculo de este factor:

$$
\text { FCpre }=1-(h l / h t)
$$

Donde:

$\mathrm{hl}=$ Horas de lluvia limitantes por año.

ht $=$ Horas al año que el Jardín Botánico está abierto.

Para identificar las horas de lluvia limitantes al año se utilizó información hora a hora desde mayo 2008 a mayo 2009 de la Estación UTP de la Red Hidroclimatológica del municipio de Pereira. Con dicha información clasificada en promedios por hora de acuerdo al mes y teniendo en cuenta las horas de prestación de los servicios del bosque del Jardín Botánico se hizo un análisis mediante el software SPSS bajo windows.

\section{Factor de Corrección Accesibilidad (FCacc)}

Mide el grado de dificultad que podrían tener los visitantes para desplazarse por el sendero, esto se midió tomando como referente la longitud e inclinación de la pendiente (Factor LS). La Tabla 1 expone los rangos de LS que suponen dificultad alta, media y baja para la accesibilidad:

Tabla 1. Clasificación del Factor LS.

\begin{tabular}{|c|c|}
\hline $\begin{array}{c}\text { Valor de LS de } \\
\text { segmentos de c/u de } \\
\text { los senderos }\end{array}$ & $\begin{array}{c}\text { Cualificación } \\
\text { dificultad para el } \\
\text { acceso por el } \\
\text { sendero }\end{array}$ \\
\hline$<2$ & Baja \\
\hline $2-20$ & Medio \\
\hline$>20$ & Alta \\
\hline
\end{tabular}

Fuente: elaboración propia con base en Montenegro, H.; Malagón, D. y Guerrero, L. (1990).

Los tramos que poseen un grado de dificultad medio o alto son los únicos considerados significativos al momento de establecer restricciones de uso. Puesto que un grado alto representa una dificultad mayor que un grado medio, se debe incorporar un factor de ponderación de 1 para el grado medio de dificultad, y de 1,5 para el alto; sin embargo, en el presente caso solo se tuvieron valores de LS medios, por lo que no se necesitó aplicar los factores de ponderación mencionados.

La siguiente es la ecuación a utilizar:

$$
F \text { Cacc }=1-(\mathrm{mm} / \mathrm{mt})
$$


Donde:

$\mathrm{mm}=$ metros de sendero con dificultad media.

$\mathrm{mt}=$ metros totales de sendero.

Se aplicó la siguiente ecuación:

$$
C C T=C C F *(F C s o c * \text { FCpre } * \text { FCacc })
$$

Cálculo de Capacidad de Carga Ecosistémica (CCE)

Para este cálculo se sometió la CCT a una serie de factores de corrección de índole ecosistémica que se explican a continuación:

\section{Factor de Corrección Erodabilidad (FCero)}

La fórmula mediante la cual se estima el FCero es la siguiente:

$$
\text { FCero }=1-(m p e / m t)
$$

Donde:

$\mathrm{mpe}=$ metros de sendero con problemas de erodabilidad.

$\mathrm{mt}=$ metros totales de sendero.

Así, para establecer los tramos de sendero $(\mathrm{m})$ con problemas de erodabilidad en el JB-UTP, se desarrollaron cálculos de pérdida de suelo basados en la Ecuación Universal de Pérdida de Suelo Revisada-RUSLE. Debido a que lo ideal era tener datos diferenciados por sendero, se partió de la obtención de datos de superficie lineal para cada uno de ellos relacionados con los cambios de pendiente; con ello se pudieron determinar factores de la RUSLE tales como Longitud e Inclinación de la Pendiente y Cobertura del Suelo en valores diferenciados por tramos y senderos.

No obstante, para los factores de la RUSLE tales como Erosividad de Lluvia y Erodabilidad solo se pudieron estimar datos generalizados para el JB, teniendo en cuenta la información con que se contaba.

\section{Factor de Corrección Afectación a Fauna (FAfau)}

El cálculo de este factor estuvo encaminado no a la restricción del número de visitantes permitidos en el Jardín Botánico, tal como se planteó con los demás factores, sino que con éste se pretendió otorgar un criterio para la planificación de las actividades que impliquen manejo de visitantes, ya sea generando ciertas restricciones o controlando determinados comportamientos, que permitan en este sentido, la recuperación y/o estabilidad natural del ecosistema.

Debido a que no existe información de línea base que analice los impactos que se derivan de la visitación al Jardín sobre la fauna o el ecosistema, se partió de la elaboración de unas encuestas, las cuales se aplicaron a los intérpretes ambientales o guías del JB-UTP, siendo estos actores clave en la observación de posibles perturbaciones o cambios ecológicos durante y/o después de las actividades que lideran.

La encuesta tiene contenidos a partir de los cuales se puede concluir cualitativamente sobre el avistamiento de fauna durante y/o después de las actividades que impliquen visitación al Jardín Botánico tales como: recorridos de interpretación ambiental, deporte aventura, vacaciones recreativas y eventos de entrada masiva al JB-UTP. Cabe resaltar el motivo por el cual se contempla el avistamiento de fauna, pues aunque éste es un criterio que aborda superficialmente el grado de perturbaciones ecológicas que puede existir, en un principio y a falta de información más completa, puede ser de gran utilidad para generar ciertas estimaciones necesarias en el presente estudio.

Se aplicó la siguiente fórmula: 


\section{$C C E=C C T *($ FCero $*$ FCAfau $)$}

\section{Cálculo de Capacidad de Gestión (CG)}

Se realizó el cálculo de la Capacidad de Gestión, mediante la aplicación de cuestionarios a dos de los funcionarios administrativos del Jardín Botánico para que evaluaran de acuerdo con su experiencia y conocimientos (Director y Coordinador Científico); ello con el fin de obtener información sobre el manejo del Jardín teniendo en cuenta cada uno de los procesos de la gestión, a saber: planeación, ejecución y control, otorgando una valoración cuantitativa y cualitativa a cada uno de ellos, así como a sus ámbitos y criterios. Los valores otorgados fueron tres (3), dos (2) y uno (1), con cualificaciones alto, medio y bajo respectivamente, los cuales fueron promediados posteriormente.

La siguiente fue la fórmula utilizada para calcular la Capacidad de Gestión.

$$
C G=(\% \text { CPlan }+\% \text { CEjec }+\% \text { CCont }) / 3
$$

Donde:

CG = Capacidad de Gestión.

CPlan = Capacidad de Planeación.

CEjec $=$ Capacidad de Ejecución .

CCont $=$ Capacidad de Control .

A continuación se exponen cada uno de los niveles de gestión que se pueden manifestar en la evaluación:

- Nivel I. Manejo insatisfactorio $(\leq 35 \%)$.

Una puntuación total menor o igual al 35\% del óptimo, indica que el área carece de los recursos mínimos necesarios para su manejo básico y, por lo tanto, no existen garantías para su permanencia a largo plazo. Los objetivos del área no podrán ser alcanzados bajo esas circunstancias.

- $\quad$ Nivel II. Manejo poco satisfactorio (36-50\%).

Una puntuación dentro de este rango, permite decir que el área posee ciertos recursos y medios que son indispensables para su manejo, pero que le faltan muchos elementos para alcanzar un nivel mínimo aceptable. Tales características imponen al área una condición de alta vulnerabilidad a la incidencia de factores coyunturales externos o internos $y$, consecuentemente, no garantizan su permanencia a largo plazo. Los objetivos del área difícilmente podrían ser alcanzados, en especial algunos objetivos primarios.

- Nivel III. Manejo medianamente satisfactorio (51-75\%).

El área dispone de los elementos mínimos para el manejo, pero presenta deficiencias esenciales que no permiten establecer una sólida base para que este manejo sea efectivo. Hay un cierto desequilibrio o desarticulación entre los ámbitos que influyen en el manejo que puede comprometer la integridad de los recursos, y el cumplimiento de objetivos podría ser solo parcial, pudiendo desatenderse sobre todo algunos de los objetivos secundarios.

- $\quad$ Nivel IV. Manejo satisfactorio (76-89\%).

Los factores y medios que posibilitan el manejo están siendo atendidos adecuadamente. Las actividades necesarias se desarrollan normalmente y con buenos resultados. La permanencia del área estaría garantizada por cuanto hay un equilibrio dinámico entre todos los ámbitos del manejo; todo el conjunto tiende normalmente hacia el cumplimiento de los objetivos de manejo.

- Nivel V. Manejo muy satisfactorio ( $\geq 90 \%)$. 
El área cuenta con todos los medios para un manejo eficiente conforme las demandas del presente. Por esto, tiene posibilidades de absorber ciertas exigencias del futuro, sin comprometer la conservación del recurso. El cumplimiento de los objetivos del área estaría garantizado.

Así, la Capacidad de Carga Ambiental (CCA) se calculó de acuerdo con la siguiente fórmula:

$$
C C A=C C E \times C G
$$

Donde:

CCA = Capacidad de Carga Ambiental

CCE = Capacidad de Carga Ecosistémica

CG = Capacidad de Gestión.

Finalmente, la CCA obtenida se utiliza multiplicándose por el NV de cada uno de los circuitos, para obtener la Capacidad de Carga Ambiental en unidades de visitantes/día para cada circuito respectivo.

\section{RESULTADOS Y DISCUSIÓN}

\section{Capacidad de Carga Turística (CCT)}

La Capacidad de Carga Física difiere en todos los circuitos de interpretación ambiental dados los datos de espacio y tiempo diferenciados para cada uno, tal como se puede ver en la Tabla 2.

No obstante, si bien cada circuito viene con una CCF diferente, los criterios para determinar los Factores de Corrección Social y de Precipitación son iguales para todos los circuitos, a saber: número de personas máximo en cada grupo, distancia entre cada grupo y horas de mayor lluvia en el JB; por lo que consecuentemente estos factores de corrección son iguales. Mientras que el Factor de Corrección por Accesibilidad sí difiere, puesto que éste va a depender de los datos de longitud e inclinación de la pendiente relativa a cada uno de ellos, sobre los que se pueda inferir dificultades medias y/o altas para el acceso. 
Tabla 2. Datos para cálculos de CCT de cada circuito en el JB-UTP.

\begin{tabular}{|c|c|c|c|c|}
\hline \multirow{2}{*}{ Parámetro } & \multicolumn{4}{|c|}{ Circuito } \\
\hline & 1 & 2 & 3 & 4 \\
\hline $\begin{array}{c}\text { Horario de Visita - Hv } \\
\text { (h) }\end{array}$ & 10 & 10 & 10 & 10 \\
\hline $\begin{array}{l}\text { Tiempo de Visita - tv } \\
\text { (h-visitaivistante) }\end{array}$ & 0,50 & 1,00 & 1,50 & 2,00 \\
\hline $\begin{array}{c}\text { Superficie - S } \\
\text { (m) }\end{array}$ & 731,5 & 1337,2 & 1487,6 & 1197,2 \\
\hline $\begin{array}{c}\text { Superficie usada por } \\
\text { persona - Sp } \\
\text { (m) }\end{array}$ & 1 & 1 & 1 & 1 \\
\hline $\begin{array}{c}\text { NV } \\
\text { (visit asidiaivisitante) }\end{array}$ & 20 & 10 & 7 & 5 \\
\hline $\begin{array}{c}\text { CCF } \\
\text { (viskasidia) }\end{array}$ & 14630 & 13372 & 9917 & 5986 \\
\hline $\begin{array}{l}\text { Núm ero máx. de } \\
\text { personas por grupo } \\
\text { (personas) }\end{array}$ & 26 & 26 & 26 & 26 \\
\hline $\begin{array}{c}\text { Distancia entre grupos } \\
\text { (m) }\end{array}$ & 56 & 56 & 56 & 56 \\
\hline $\begin{array}{c}\text { NG } \\
\text { (No. grupos } \\
\text { sim ultáneamente en cada } \\
\text { circuito) }\end{array}$ & 13 & 24 & 27 & 21 \\
\hline $\begin{array}{c}\text { P } \\
\text { (No. personas } \\
\text { grupoicircuito) }\end{array}$ & 340 & 621 & 691 & 556 \\
\hline mIFCsoc & 391,88 & 716,36 & 796,93 & 641,36 \\
\hline FCsoc & 0,46 & 0,46 & 0,46 & 0,46 \\
\hline $\begin{array}{c}\text { Horas de lluvia limitantes } \\
\text { al an̂́o - hl } \\
\text { (h) }\end{array}$ & 365 & 365 & 365 & 365 \\
\hline $\begin{array}{c}\text { Horas anuales en que el } \\
\text { J日 está abierto - ht } \\
\text { (h) }\end{array}$ & 3650 & 3650 & 3650 & 3650 \\
\hline FCpre & 0,90 & 0,90 & 0,90 & 0,90 \\
\hline $\begin{array}{l}\text { Long. sendero con } \\
\text { díniculad media para ia } \\
\text { accesibiliad } \mathrm{mm} \\
\text { ( } \mathrm{m})\end{array}$ & 247,10 & 487,40 & 487,40 & 448,80 \\
\hline FCaCC & 0,66 & 0,64 & 0,67 & 0,63 \\
\hline
\end{tabular}

Capacidad de Carga Ecosistémica (CCE)

Factor de Corrección Erodabilidad (FCero)

Tabla 3. Datos para cálculo de CCE de cada circuito en el JB-UTP. 


\begin{tabular}{|c|c|c|c|c|}
\hline \multirow{2}{*}{ Parámetro } & \multicolumn{4}{|c|}{ Circuito } \\
\cline { 2 - 5 } & $\mathbf{1}$ & $\mathbf{2}$ & $\mathbf{3}$ & $\mathbf{4}$ \\
\hline $\begin{array}{c}\text { Long. Sendero } \\
\text { con } \\
\begin{array}{c}\text { susceptibilidad } \\
\text { media a la } \\
\text { erosión } \\
\text { (m) }\end{array}\end{array}$ & 322,60 & 537,90 & 537,90 & 514,80 \\
\hline $\begin{array}{c}\text { FCero } \\
\text { CCT }\end{array}$ & 0,56 & 0,60 & 0,64 & 0,57 \\
\hline $\begin{array}{c}\text { Capacidad de } \\
\text { Carga }\end{array}$ & $\mathbf{2 2 6 3}$ & $\mathbf{2 1 2 3}$ & $\mathbf{1 7 7 9}$ & $\mathbf{8 9 1}$ \\
$\begin{array}{c}\text { Ecosistémica } \\
\text { (visitas/día) }\end{array}$ & & & & \\
\hline
\end{tabular}

No fue posible calcular un Factor de Corrección Afectación a Fauna ya que existe una carencia de información objetiva para este caso, y por ello no se puede determinar un índice como tal para aplicarle a la Capacidad de Carga Turística. Sin embargo, los resultados de las encuestas realizadas (Tabla 4) son, sin duda alguna, de importancia para justificar la necesidad de evaluaciones y/o monitoreos respectivos.

Factor de Corrección Afectación a Fauna (FAfau)

\begin{tabular}{|c|c|c|c|c|c|c|c|c|c|}
\hline \multirow{3}{*}{\multicolumn{2}{|c|}{$\begin{array}{c}\text { Activided an al } \\
\text { JE.UTP }\end{array}$}} & \multicolumn{8}{|c|}{ Avictemisnto de toune } \\
\hline & & \multicolumn{4}{|c|}{ El mismo dis de le sethided } & \multicolumn{4}{|c|}{ Diec decpuec de la settrided } \\
\hline & & satictactorid & \begin{tabular}{|l|} 
Nodlenemerts \\
catictactorio
\end{tabular} & \begin{tabular}{|l|} 
Incetictectoria \\
\end{tabular} & \begin{tabular}{|c|} 
No \\
cesbeiNo \\
responsts \\
\end{tabular} & \begin{tabular}{|l|} 
astictactorb \\
\end{tabular} & \begin{tabular}{|} 
Nadlenamerte \\
catictectorlo
\end{tabular} & \begin{tabular}{|l|} 
Incstictsctorio \\
\end{tabular} & $\begin{array}{l}\text { No } \\
\text { cabeiNo } \\
\text { recponds }\end{array}$ \\
\hline \multirow{2}{*}{88} & \begin{tabular}{|l|}
$1-5$ \\
Vist:ess
\end{tabular} & $50 \%$ & $50 \%$ & $0 \%$ & $0 \%$ & $62 \%$ & $25 \%$ & $0 \%$ & $13 \%$ \\
\hline & 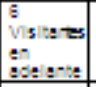 & $12 \%$ & $38 \%$ & $50 \%$ & $0 \%$ & $25 \%$ & $37 \%$ & $38 \%$ & $0 \%$ \\
\hline \multirow{2}{*}{ 造题 } & \begin{tabular}{|l|}
$1-5$ \\
Usiatos
\end{tabular} & $37 \%$ & $38 \%$ & $25 \%$ & $0 \%$ & $37 \%$ & $25 \%$ & $25 \%$ & $13 \%$ \\
\hline & 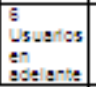 & $0 \%$ & $50 \%$ & $50 \%$ & $0 \%$ & $12 \%$ & $38 \%$ & $50 \%$ & $0 \%$ \\
\hline \multicolumn{2}{|c|}{$\begin{array}{l}\text { Vacsolones } \\
\text { feccestives }\end{array}$} & $25 \%$ & $50 \%$ & $12 \%$ & $13 \%$ & $12 \%$ & $50 \%$ & $25 \%$ & $13 \%$ \\
\hline \multicolumn{2}{|c|}{ 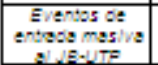 } & $12 \%$ & $12 \%$ & $63 \%$ & $13 \%$ & $12 \%$ & $37 \%$ & $38 \%$ & $13 \%$ \\
\hline
\end{tabular}

Recorridos de interpretación ambiental

Se puede evidenciar claramente que el avistamiento de fauna oscila con el mismo peso, entre los rangos satisfactorio y medianamente satisfactorio, cuando se manejan grupos entre 1-5 visitantes el mismo día de la actividad; sin embargo, ya días después de realizada la actividad se observa que al manejar grupos de igual número es más satisfactorio el avistamiento de fauna, indicándose posiblemente con ello una recuperación de alguna u otra forma del ecosistema respecto a las perturbaciones que hubieran podido ser ocasionadas. De otro lado, se tiene que el $13 \%$ califica el ítem "No sabe/No responde", demostrándose de esta forma la necesidad de realizar observaciones planificadas y rigurosas sobre este aspecto que brinden información más completa para su posterior análisis.

En lo referente al manejo de grupos de 6 visitantes en adelante, el proceso de avistamiento de fauna comienza a calificarse de una manera muy distribuida entre todos los rangos posibles, desde "Satisfactorio" hasta "No sabe/No responde", aunque cabe hacer un énfasis en que el proceso se torna, según los intérpretes ambientales encuestados, más insatisfactorio durante el mismo día de la actividad.

\section{Deporte Aventura}


Cuando se manejan grupos pequeños el proceso se califica de manera distribuida entre los rangos. En el caso de grupos grandes se tiene, tanto en el mismo día como días después de la actividad, una tendencia mayor entre los rangos "Medianamente satisfactorio" e "Insatisfactorio".

\section{Vacaciones Recreativas}

Se presenta una tendencia muy distribuida en las calificaciones que dan los intérpretes ambientales.

\section{Eventos de entrada masiva}

Si bien se observa una tendencia de igual forma distribuida, existen calificaciones mayores para los rangos entre "Medianamente satisfactorio" e "Insatisfactorio".

En general, es de resaltar que la forma tan distribuida de las calificaciones expresa una falta de consenso sobre las posibles perturbaciones que se puedan generar en el avistamiento de fauna en el JB-UTP, y por ende se afirma la necesidad ya mencionada de ejecutar observaciones sobre este aspecto con cierta rigurosidad y que sean previamente programadas, esto con el fin de obtener información completa que posibilite un análisis de mayor objetividad.

\section{Capacidad de Gestión (CG)}

El valor de CG refleja un Nivel de Gestión IV, lo cual implica un manejo satisfactorio, es decir porcentajes obtenidos respecto a la evaluación que oscilan entre $76-89 \%$ (ver Tabla 5). En este sentido cabe decir que en el JB-UTP:

- Los factores y medios que posibilitan el manejo están siendo atendidos adecuadamente.

- Las actividades necesarias se desarrollan normalmente y con buenos resultados.

- La permanencia del área estaría garantizada por cuanto hay un equilibrio dinámico entre todos los ámbitos del manejo; todo el conjunto tiende normalmente hacia el cumplimiento de los objetivos de manejo.

Tabla 5. Datos de Capacidad de Gestión en el JB-UTP.

\begin{tabular}{|c|c|c|c|}
\hline \multirow{2}{*}{ Parámetro } & \multicolumn{3}{|c|}{ Circuito } \\
\cline { 2 - 4 } & $\mathbf{1}$ & 2 & \multicolumn{3}{|c|}{$\mathbf{3}$} & $\mathbf{4}$ \\
\hline $\begin{array}{c}\text { CPlan } \\
\text { Capacidad de } \\
\text { Planeacón } \\
\text { (\%) }\end{array}$ & \multicolumn{3}{|c|}{$76,67 \%$} \\
\hline $\begin{array}{c}\text { CEjec } \\
\text { Capacidad de } \\
\text { Ejecución } \\
\text { (\%) }\end{array}$ & \multicolumn{2}{|c|}{$83,11 \%$} \\
\hline $\begin{array}{c}\text { CCont } \\
\text { Capacidad de } \\
\text { Contro } \\
\text { (\%) }\end{array}$ & $61,67 \%$ \\
\hline $\begin{array}{c}\text { CG } \\
\text { Capacidad de } \\
\text { Gestión } \\
\text { (\%) }\end{array}$ & \multicolumn{2}{|c|}{} \\
\hline
\end{tabular}

Fuente: elaboración propia.

\section{Capacidad de Carga Ambiental (CCA)}

Para el cálculo de la CCA se tuvo en cuenta el menor valor de CCE, ya que éste es el más restrictivo. En este caso el valor que correspondió tomar fue el del Circuito 4 (891 visitas/día). 
Finalmente, tal como se puede observar en la Tabla 6, el valor de Capacidad de Carga Ambiental diaria promedio para el JB-UTP es de 82 visitantes/día, para un promedio anual de 30.017 visitantes/año.

Tabla 6. Cálculo de la Capacidad de Carga Ambiental en el JB-UTP.

\begin{tabular}{|c|c|c|c|c|}
\hline \multirow{2}{*}{ Parámetro } & \multicolumn{4}{|c|}{ Circuito } \\
\hline & 1 & 2 & 3 & 4 \\
\hline $\begin{array}{c}\text { CCA } \\
\text { Capacidad de Carga } \\
\text { Ambiental } \\
\text { (Msitasidia) }\end{array}$ & \multicolumn{4}{|c|}{658} \\
\hline $\begin{array}{c}\text { CCA } \\
\text { Capacidad de Carga } \\
\text { Ambiental } \\
\text { (visistantes/dia) }\end{array}$ & 33 & 66 & 99 & 132 \\
\hline $\begin{array}{c}\text { CCA } \\
\text { Capacidad de Carga } \\
\text { Ambiental } \\
\text { (uisitantesknôno) }\end{array}$ & 12007 & 24013 & 36020 & 48027 \\
\hline $\begin{array}{c}\text { CCA } \\
\text { Capacidad de Carga } \\
\text { Ambiental } \\
\text { (visitantesidia } \\
\text { Promedio J日-UTP) }\end{array}$ & \multicolumn{4}{|c|}{82} \\
\hline $\begin{array}{c}\text { CCA } \\
\text { Capacidad de } \\
\text { Carga Ambiental } \\
\text { [visitanteskñó } \\
\text { Promedo JB-UTP ] }\end{array}$ & \multicolumn{4}{|c|}{30017} \\
\hline
\end{tabular}

Fuente: elaboración propia.

\section{CONCLUSIONES}

- Si bien los Jardines Botánicos no son áreas protegidas como tales, sí son aquellas en las cuales se llevan a cabo procesos similares de conservación y de manejo ecoturístico. Por lo tanto, los estudios de capacidad de carga ambiental juegan un papel indispensable en el establecimiento de criterios objetivos para sustentar su manejo.

- Aunque la metodología se trabaja estructuralmente alrededor de la "Determinación de la Capacidad de Carga Turística", es posible observar que se hizo un intento por tratar conceptos más integrales, que respondieran realmente a un enfoque ambiental del manejo del área; es decir, teniendo en cuenta aspectos relacionados con la resiliencia ecosistémica, con la satisfacción del visitante y con la capacidad institucional para la gestión en el Jardín Botánico.

- Respecto a la evaluación de la capacidad de gestión del Jardín Botánico, se puede decir que de igual manera si bien se adoptó una estructura de evaluación similar a la propuesta desde la metodología de "Capacidad de Carga Turística", es de resaltar el significado más integral que se le quiso dar a ésta, comprendiéndola a partir de los tres procesos básicos de la administración: planeación, ejecución y control.

- Ya que la presente determinación de la capacidad de carga ambiental no tendría sentido si el esquema trabajado no posibilitara futuras actualizaciones, se consideró una justificación teórico-conceptual del proceso monitoreo, así como una flexibilidad metodológica que permitiera el ajuste.

- Es primordial concebir los estudios de capacidad de carga ambiental no solo desde una contextualización teórico-conceptual y metodológica, sino a su vez teniendo en cuenta los aspectos político-normativos que los sustentan, en este caso: Ley de Turismo y Política para el Desarrollo del Turismo.

- Es importante abordar cualquier estudio de carácter ambiental contemplando inicialmente una diagnosis general que aborde aspectos tanto organizativos y de infraestructura, así como biofísicos y socioculturales. Esto es indispensable a la hora de determinar las relaciones ambientales que configuran el área objeto de estudio, y con ello lograr una mayor comprensión de los fenómenos que allí se dan, en este caso lo relativo a la visitación y los impactos tanto positivos como negativos que se generan. 
- Fue necesario idear una manera como la metodología de "Capacidad de Carga Turística" conservando su estructura, se acomodara a la conceptualización ambiental que se quería imprimir en el presente estudio, por ello se establecieron: en primer lugar una fase turística, luego una ecosistémica y finalmente teniendo en cuenta la capacidad de gestión, una fase ambiental que relacionara las anteriores.

- Gracias a la información y herramientas con que se contaba, se pudo dar un análisis mucho más completo de los Factores de Corrección por Accesibilidad, Precipitación y Erodabilidad, posibilitando con ello concepciones más objetivas de estos aspectos dentro del proceso de determinación de la "Capacidad de Carga Ambiental".

- La afectación a fauna fue un factor de corrección que no se pudo determinar debido principalmente a las limitaciones en información; no obstante, las encuestas realizadas a los intérpretes ambientales fueron un insumo valioso para generar algunas conclusiones y dar soporte a la necesidad de monitoreos rigurosos sobre este proceso.

- La Evaluación de la Capacidad de Gestión del JB-UTP se convierte en un instrumento de gran utilidad no solo para el presente estudio en lo relacionado con los aspectos de manejo del área, sino también para dar sustento a medidas de mejoramiento que lleven a que la gestión sea cada vez más óptima y se puedan cumplir los objetivos misionales del Jardín Botánico.

- Dado que la Evaluación de la Capacidad de Gestión del JB-UTP brinda una especie de diagnóstico del JB-UTP en materia de administración, es bien importante tener en cuenta el estado detallado de los criterios que conforman según el esquema un proceso efectivo de planeación, ejecución y/o control, pues a partir de éstos se pueden establecer medidas concretas de mejoramiento.

- Los análisis sobre el suelo del Jardín Botánico realizados no sirven únicamente para el presente estudio, sino que de la misma forma pueden ser de importancia para cualquier otro tipo de estudio que necesite datos e información sobre el medio edáfico en el JB-UTP.

- La información cuantitativa de la "Capacidad de Carga Ambiental" se presenta para cada circuito de interpretación ambiental, dadas las características propias de cada uno y sus diferencias, marcadas estas últimas principalmente por la longitud y duración de los recorridos. Sin embargo, para el cálculo final (en el sentido general del JB-UTP) se tiene en cuenta el menor valor de CCE, pues éste por su carácter es el que genera la mayor restricción para la entrada de visitantes.

- En lo referente al proceso de planeación en el JB-UTP se tiene que los "procesos diagnósticos", "roles y actores" y "horizontes de ejecución - coherencia entre fines y medios" son los ámbitos que más requieren de medidas de mejoramiento.

- En términos de ejecución, la evaluación realizada indica que el ámbito "profesional, comunitario e institucional" es el que más necesita de ajustes para su optimización principalmente en lo referente a la "capacidad técnico-operativa", "actitudes del personal del área" y "apoyo y participación comunitaria".

- Otros criterios dentro de la evaluación de la capacidad de ejecución en el JB-UTP, que individualmente requieren de medidas de mejoramiento, son la "consecución de recursos", los "incentivos al personal" y el "programa de educación ambiental".

- En lo relacionado con el control, dentro de la Evaluación de la Capacidad de Gestión en el JB-UTP, se tiene que éste en general es el proceso que mayores ajustes para su optimización demanda, siendo los ámbitos más críticos a tener en cuenta: el "monitoreo", la "veeduría social" y la "retroalimentación y ajuste".

\section{RECOMENDACIONES}

- Aunque la metodología de "Capacidad de Carga Turística" proporciona elementos básicos que permiten comprender la importancia de los estudios de capacidad de carga, ésta se puede caracterizar en ciertos momentos por ser muy lineal, tal como se dio para el cálculo del "Factor de Corrección por Afectación a Fauna"; por tal motivo, sería provechoso que en posteriores actualizaciones del presente estudio se pudiera complementar con otros aspectos metodológicos que puedan acomodarse a determinadas condiciones y/o restricciones.

- Debido a que los datos utilizados en el cálculo del Factor de Corrección por Precipitación, así como en el Subfactor Erosividad de Lluvia en el de Erodabilidad pertenecen apenas a un año de recolección (2009), es necesario ir ajustando los cálculos realizados, al proceso histórico que se vaya generando en la toma de datos climatológicos en la estación meteorológica de la UTP.

- Realizar un muestreo de suelo en el JB-UTP para determinar las propiedades físicas del mismo teniendo en cuenta el establecimiento de parcelas por cada sendero, y así con ello complementar los análisis de suelo hechos durante el presente trabajo. 
- Evaluar el Subfactor de Cobertura de Suelo dentro del Factor de Corrección por Erodabilidad, generando o empleando una clasificación que contenga variables dentro de las coberturas boscosas, y en este orden de ideas ajustar más los resultados a aplicaciones de RUSLE en áreas que son específicamente de conservación y no de producción agropecuaria.

- Ajustar los datos en la Fase de Capacidad de Carga Ecosistémica a información objetiva sobre la afectación a fauna derivada de la visitación al Jardín Botánico.

- Para próximas evaluaciones de la capacidad de gestión del Jardín Botánico tener en cuenta una metodología participativa, en donde se integren diferentes actores relacionados con el Jardín Botánico, a saber: instancias superiores de la universidad, comunidad, academia, entidades gubernamentales y no gubernamentales y personal administrativo y operativo del Jardín.

- Integrar las necesidades de información diagnóstica en el JB-UTP con las temáticas académicas de semilleros y grupos de investigación de la universidad.

- Generar un espacio para el monitoreo de los procesos de visitación, principalmente en lo relacionado con la afectación al ecosistema, vinculando a las funciones de los intérpretes ambientales una labor de observación y registro respectiva, utilizando para ello formatos determinados y avalados por el Sistema de Gestión de la Calidad de la universidad.

\section{REFERENCIAS}

- Arango, P. (2003). Determinación de la capacidad de carga y diseño de la propuesta de interpretación ambiental para el Jardín Botánico de la Universidad Tecnológica de Pereira, Pereira. Trabajo de Grado (Administradora del Medio Ambiente), Universidad Tecnológica de Pereira, Facultad de Ciencias Ambientales, Escuela de Administración Ambiental.

- Cifuentes, M.; Izurieta, A. y De Faria, H. (2000). Medición de la Efectividad de Manejo de Áreas Protegidas. Turrialba: Costa Rica. GTZ, UICN, WWF. Serie Técnica No. 2. Obtenido el 25 de julio de 2009, desde http:/lassets.panda.org/downloads/wwfcameasuringes.pdf

- Cifuentes, M.; Mesquita, C.; Méndez, J.; Morales, M.; Aguilar, N.; Cancino, D.; Gallo, M.; Jolón, M.; Ramírez, C.; Ribeiro, N.; Sandoval, E. y Turcios, M. (1999). Capacidad de carga turística de las áreas de uso público del Monumento Nacional Guayabo. Turrialba: Costa Rica. WWF-CATIE. Obtenido el 25 de julio de 2009, desde http://assets. panda.org/downloads/wwfcaguayabo.pdf

- Contraloría General de la República. (2007). Estado de los Recursos Naturales y del Ambiente 2007-2008. Bogotá, Colombia: Imprenta Nacional. Obtenido el 25 de julio de 2009, desde http://www.contraloriagen.gov.co/html/publicaciones/publicaciones_inicio.ap

- Grupo de Estudio en Fauna Silvestre - Medicina Veterinaria y Zootecnia Universidad de Caldas. (2005). Muestreo Rápido de Biodiversidad en el Jardín Botánico Universidad Tecnológica de Pereira. Pereira: Colombia. Disponible en: Archivo Digital Jardín Botánico Universidad Tecnológica de Pereira.

- Hernandez, H. (2003). Determinación de la capacidad de carga ecoturística como herramienta de planificación y manejo del Santuario de Fauna y Flora Otún Quimbaya Vereda La Suiza, Municipio de Pereira, Pereira. Trabajo de Grado (Administrador del Medio Ambiente), Universidad Tecnológica de Pereira, Facultad de Ciencias Ambientales, Escuela de Administración Ambiental.

- Jardín Botánico y Campus Universidad Tecnológica de Pereira. (2005). Index Plantarum, 1. Pereira: Colombia.

- Jardín Botánico Universidad Tecnológica de Pereira. (2007). Postulación convocatoria décimo tercer concurso distinción medio ambiente ciudad de Pereira. Pereira. Disponible en: Archivo Digital Jardín Botánico Universidad Tecnológica de Pereira.

- Ley 299 de 1996. (1996). Por la cual se protege la flora colombiana, se reglamentan los jardines botánicos y se dictan otras disposiciones. (Diario Oficial 42.845 del 26 de julio de 1996). Colombia, Ministerio del Medio Ambiente y Ministerio de Hacienda y Crédito Público. Obtenido el 25 de julio de 2009, desde http://www.alcaldiabogota.gov.co/sisjur/normas/Norma1.jsp?i=5518

- Ley 300 de 1996. (1996). Por la cual se expide la Ley General de Turismo y se dictan otras disposiciones. (Diario Oficial No. 42.845, de 30 de Julio de 1996). Colombia, Ministerio de Desarrollo Económico. Obtenido el 25 de julio de 2009, desde http://www.mincomercio.gov.co/econtent/documentos/turismo/ Normatividad/leyes/Ley_300_1996.pdf

- Montenegro, H.; Malagón, D. y Guerrero, L. (1990). Propiedades Físicas de los Suelos. Bogotá: Colombia. Instituto Geográfico Agustín Codazzi-IGAC. 
- Plan Nacional para Jardines Botánicos de Colombia (Documento Borrador). (1998). Ministerio del Medio Ambiente, Instituto de Investigaciones Biológicas Alexander von Humboldt y la Red Nacional de Jardines Botánicos de Colombia. Bogotá: Colombia.

- Política para el Desarrollo del Ecoturismo. (2003). Ministerio de Comercio, Industria y Turismo y Ministerio de Ambiente, Vivienda y Desarrollo Territorial. Bogotá: Colombia. Obtenido el 25 de julio de 2009, desde http://www.mincomercio.gov.co/econtent/Documentos/turismo/2005/ Publicaciones/PoliticaEcoturismo.pdf

- Quintana, R. (2005). Bases para la implementación de un sistema de gestión ambiental para el Jardín Botánico Universidad Tecnológica de Pereira, Pereira. Trabajo de Grado (Administrador del Medio Ambiente), Universidad Tecnológica de Pereira, Facultad de Ciencias Ambientales, Escuela de Administración Ambiental.

- Rome, A. (1999). Monitoreo de los impactos turísticos: Un examen de metodologías y recomendaciones para el desarrollo de programas de monitoreo en América Latina. En Serie Informes Técnicos Ecoturismo Número, 1. Obtenido el 25 de julio de 2009, desde http://www.parksinperil.org/files/d4eecotourismimpactsmonitoringspa.pdf

- Wyse, P.S. y Sutherland L.A. (2000). Agenda Internacional para la Conservación en Jardines Botánicos. U.K.: Organización Internacional para la Conservación en Jardines Botánicos - $\mathrm{BGCl}-$.

1. Director Jardín Botánico Universidad Tecnológica de Pereira. Administrador del Medio Ambiente. Magíster en Investigación de Operaciones.

2. Joven Investigadora Jardín Botánico Universidad Tecnológica de Pereira. Administradora del Medio Ambiente.

3. De acuerdo a indagaciones realizadas en Internet en la página de los grupos de investigación de Colciencias, aparece como uno de los proyectos desarrollados por el Jardín Botánico José Celestino Mutis un estudio de capacidad de carga.

4. Ver conceptualización de Augusto Ángel Maya sobre la relación ecosistema-cultura. [Ángel, A (1992). Ecosistema y Cultura. En Cuadernos Ambientales, 3. Colombia: Ministerio de Educación Nacional].

5. Estos supuestos fueron resultado de un consenso con la Coordinación de Educación Ambiental del Jardín Botánico Universidad Tecnológica de Pereira. 